\title{
Response of Xerotolerant and Non-tolerant Yeasts to Water Stress
}

\author{
By MARGARET EDGLEY AND A. D. BROWN \\ Department of Biology, University of Wollongong, Wollongong, \\ New South Wales 2500, Australia
}

(Received 19 August 1977)

\section{INTRODUCTION}

Brown \& Simpson (1972) reported the general accumulation of arabitol by xerotolerant yeasts, but not by non-tolerant species, when they were grown in a 'normal' dilute medium, i.e. a medium with a high level of thermodynamically available water. Arabitol was accredited with the role of compatible solute in the water relations of the tolerant species. Brown (1974) reported an increase in the polyhydric alcohol content of a xerotolerant yeast, Saccharomyces rouxii, when grown in media in which the thermodynamic water activity $\left(a_{\mathrm{w}}\right)$ was lowered by polyethylene glycol or by glucose. The implication was that the increased polyol content could be attributed to arabitol. We report here that arabitol content does not respond appreciably to changes in water activity, that the changed polyol content can be attributed to glycerol and that the non-tolerant species, Saccharomyces cerevisiae, also responds to diminished water activity by accumulating glycerol. There are, however, important physiological differences between the two species in the mechanism of accumulation. The essential findings have been summarized elsewhere (Brown, 1977). The present paper contains experimental details in support of that summary.

\section{METHODS}

The yeasts, Saccharomyces rouxii (strain YA, Anand \& Brown, 1968; xerotolerant) and Saccharomyces cerevisiae (strain Y41, Anand \& Brown, 1968; non-tolerant), were maintained and grown essentially as described by Anand \& Brown (1968) in media adjusted to the specified water activity with sodium chloride or polyethylene glycol (mol. wt 200). The following inoculation schedule was used. A liquid 'preinoculum' (basal medium, $a_{\mathrm{w}} 0.997,50 \mathrm{ml}$ ) was inoculated by loop from the stock culture on an agar slope. The preinoculum was incubated at $30^{\circ} \mathrm{C}$ with rotary agitation for about $24 \mathrm{~h}$. An 'inoculum' (50 or $100 \mathrm{ml}$ fresh medium at the required experimental water activity) was then seeded with a $5 \%(\mathrm{v} / \mathrm{v})$ transfer from the preinoculum and incubated as described above for about $15 \mathrm{~h}$. This culture was used to inoculate the experimental culture; a $10 \%$ inoculum was used. Unless specified otherwise, the yeasts were harvested in mid-exponential growth phase. Before harvesting, the cultures were chilled to about $0{ }^{\circ} \mathrm{C}$ and the yeasts were collected by centrifugation in the cold. They were washed twice with cold water (about $5 \mathrm{ml}$ per 50 to $100 \mathrm{mg}$ yeast) in the centrifuge; the washings were combined, concentrated under vacuum and freeze-dried.

Polyhydric alcohols were determined in the culture supernatant, if necessary after concentration, and in the washings and extracts prepared as above. Intracellular polyol was defined as the polyol contained in the washings of the whole yeast plus the polyol extracted from the freeze-dried yeast. Polyols were identified by paper chromatography (Brown \& Simpson, 1972). They were estimated by quantitative chromatography on 3MM paper in the same solvent system, followed by elution with water from the chromatogram and estimation by the periodate oxidation method of Hanahan \& Olley (1958). Glucose was estimated with glucose oxidase according to Davies \& Wayman (1973). The 'dry mass' of yeast used in the calculation of polyol yield was the mass of a freeze-dried sample, prepared as above, before extraction. 


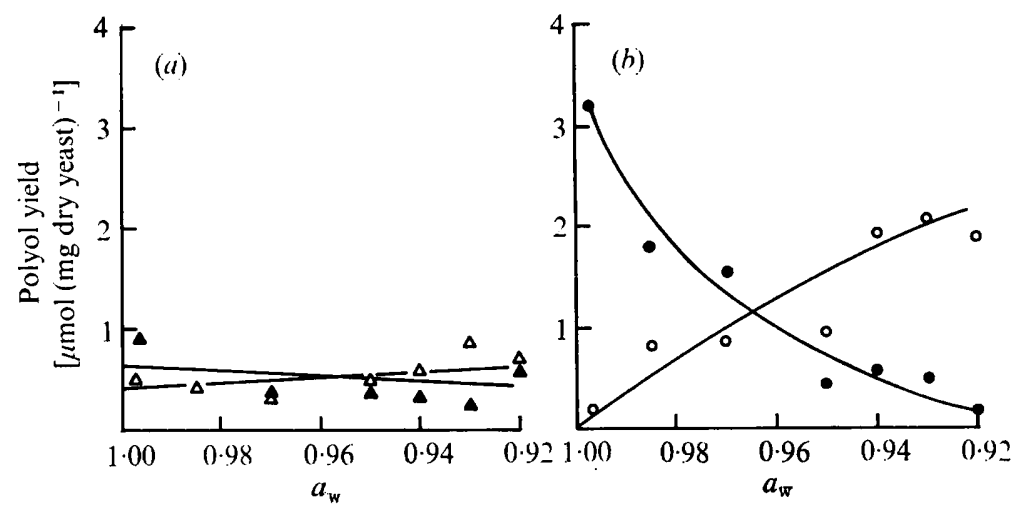

Fig. 1. The distribution of arabitol (a) and glycerol $(b)$ in Saccharomyces rouxii in response to water activity adjusted in growth media with polyethylene glycol (mol. wt 200). The yeast was harvested in mid-exponential growth phase. $\Delta$, Intracellular arabitol; $\boldsymbol{\Delta}$, extracellular arabitol; $\bigcirc$, intracellular glycerol; 0 , extracellular glycerol.

\section{RESULTS AND DISCUSSION}

Figure 1 shows polyol production (intracellular and extracellular glycerol and arabitol) by Saccharomyces rouxii as a function of water activity adjusted with polyethylene glycol (mol. wt 200). These determinations were made primarily for comparison with analogous experiments using sodium chloride to adjust water activity. The results of those experiments, which are essentially similar, have been given elsewhere (Brown, 1977). The essence of the response of $S$. rouxii to lowered water activity was to retain within the cell an increasing proportion of glycerol, the total amount of which did not change greatly with water activity. Arabitol was not appreciably affected by water activity either in the amount synthesized or retained. Saccharomyces cerevisiae responded to diminishing water activity by synthesizing much more glycerol but retaining a lower and less variable proportion of it. At approximately mid-exponential growth phase in basal medium $\left(a_{\mathrm{w}} 0.997\right)$ duplicate experiments showed $S$. cerevisiae to contain 0.06 and $0.08 \mu \mathrm{mol}$ glycerol (mg dry yeast) ${ }^{-1}$ whereas the medium contained the equivalent of 4.5 and $5.4 \mu \mathrm{mol}$ glycerol (mg dry yeast) ${ }^{-1}$. At $a_{\mathrm{w}} 0.94$ (adjusted with polyethylene glycol) the corresponding values were 1.6 and $2.5 \mu \mathrm{mol}$ (mg dry yeast $^{-1}$ for intracellular glycerol and 7.8 and $22.2 \mu$ mol (mg dry yeast) ${ }^{-1}$ for extracellular glycerol. These results show essentially the same trend as the more detailed analyses reported elsewhere (Brown, 1977) for growth media adjusted with sodium chloride. A physiological consequence of these response patterns is apparent when the polyol yields are converted to concentrations and considered as 'retention factors' (internal concentration/external concentration). Over the range of $a_{\mathrm{w}}$ values from 0.997 to 0.92 , retention factors for $S$. rouxii corresponding to Fig. 1 increased from about 100 to 8000 for glycerol and remained at about 100 for arabitol. The corresponding values in media adjusted with sodium chloride over the range of $a_{\mathrm{w}}$ values from 0.997 to 0.873 were about 150 to 8000 for glycerol and between 350 and 400 for arabitol. The retention factor for glycerol in $S$. cerevisiae increased from about 13 at $a_{w} 0.997$ to between 240 and 340 for the range of $a_{w}$ values from 0.97 to 0.94 . With sodium chloride, glycerol retention by this species also increased on the initial lowering of water activity from 0.997 and thereafter remained about constant. The retention factor at $a_{\mathrm{w}}$ 0.997 was about 55 and, from $a_{\mathrm{w}} 0.982$ to 0.936 , it was of the order 300 to 400 .

Thus the response of $S$. rouxii to water stress was at the level of glycerol permeation/transport whereas that of $S$. cerevisiae was metabolic. Lowering the amount of thermodynamically available water forces the non-tolerant species, $S$. cerevisiae, to divert a greater proportion of its metabolic activity to glycerol production. This is accompanied by an increase in the amount of glucose consumed by the yeast during growth. For example, $S$. rouxii, 
harvested in early- to mid-exponential growth phase $\left(0.4\right.$ to $1.3 \mathrm{mg}$ dry yeast $\left.\mathrm{ml}^{-1}\right)$, had consumed glucose up to that point in amounts within a range equivalent to 17 to $29 \mu \mathrm{mol}$ glucose (mg dry yeast) ${ }^{-1}$. No difference was evident between cultures in basal medium $\left(a_{\mathrm{w}} 0.997\right)$ or media adjusted to $a_{\mathrm{w}} 0.94$ with either sodium chloride or polyethylene glycol. Saccharomyces cerevisiae in early- to mid-exponential phase $\left(0.4\right.$ to $0.8 \mathrm{mg}$ dry yeast $\left.\mathrm{ml}^{-1}\right)$ was similar when cultured in basal medium, having a glucose consumption equivalent to 18 to $42 \mu \mathrm{mol}$ glucose (mg dry yeast) ${ }^{-1}$. At $a_{\mathrm{w}} 0 \cdot 94$, however, this species increased its glucose consumption to about $81 \mu \mathrm{mol}$ glucose $(\mathrm{mg} \text { dry yeast })^{-1}$ in media adjusted with sodium chloride $\left(0.3\right.$ to $0.4 \mathrm{mg}$ dry yeast $\left.\mathrm{ml}^{-1}\right)$ and to 53 to $61 \mu \mathrm{mol}$ glucose (mg dry yeast) ${ }^{-1}$ in media adjusted with polyethylene glycol $\left(0.3\right.$ to $0.5 \mathrm{mg}$ dry yeast $\left.\mathrm{ml}^{-1}\right)$. A more detailed and comprehensive representation of glucose consumption by the two yeasts in response to sodium chloride concentration has been reported by Brown (1977).

The results have two major implications for the physiology of xerotolerance in $S$. rouxii. The first is that, although arabitol can act as a 'second line' compatible solute (see Brown, 1977), the important compatible solute and major osmoregulatory substance under conditions of increasing dehydration is glycerol, as is to be expected from its enzymological effects (see Brown \& Simpson, 1972; Simpson, 1976; Brown, 1977). Although arabitol can offer a significant degree of enzyme protection in concentrated solution, it is not as effective as glycerol in that respect and its intracellular concentration is not significantly regulated by water activity. Secondly, within the water activity limits in which it can grow, $S$. cerevisiae accumulates as much glycerol as does $S$. rouxii but, in spite of that, it cannot tolerate water activities as low as can $S$. rouxii. The differences in their tolerances can be attributed at least partly to the different methods used by the two yeasts to achieve glycerol accumulation. The method used by $S$. rouxii is conservative whereas that used by $S$. cerevisiae is energetically wasteful. A limit of tolerance of the latter species is thus presumably reached when an unacceptably high proportion of total metabolic activity is diverted to glycerol production. This interpretation is consistent with results of Anand (cited by Brown, 1976) who showed that addition of glycerol to a growth medium adjusted with sucrose to a limiting level of water activity, extended the water activity tolerance range of a number of yeasts.

The results also imply that the osmoregulatory mechanism is primarily biophysical (permeation/transport) in S. rouxii and primarily metabolic in S. cerevisiae. Some aspects of the regulatory process have been discussed elsewhere (Brown, 1977).

\section{REFERENCES}

ANAND, J. C. \& Brown, A. D. (1968). Growth rate patterns of the so-called osmophilic and nonosmophilic yeasts in solutions of polyethylene glycol. Journal of General Microbiology 52, 205-212.

Brown, A. D. (1974). Microbial water relations: features of the intracellular composition of sugar-tolerant yeasts. Journal of Bacteriology 118, 769-777.

Brown, A. D. (1976). Microbial water stress. Bacteriological Reviews 40, 803-846.

Brown, A. D. (1977). Compatible solutes and extreme water stress in eukaryotic microorganisms. Advances in Microbial Physiology 17, 181-242.
Brown, A. D. \& Simpson, J. R. (1972). Water relations of sugar-tolerant yeasts: the role of intracellular polyols. Journal of General Microbiology 72, 589-591.

Davies, R. \& Wayman, F. J. (1973). Estimation of glucose and invertase activity in the presence of thiols. Analytical Biochemistry 55, 143-153.

Hanahan, D. J. \& Olley, J. N. (1958). Chemical nature of monophosphoinositides. Journal of Biological Chemistry 231, 813-828.

Simpson, J. R. (1976). Water relations of the sugartolerant yeast, Saccharomyces rouxii. Ph.D. thesis, University of New South Wales, Australia. 